\title{
Pricing weather derivatives: An application to the electrical utility
}

\author{
Zhixia Zou ${ }^{1} \cdot$ Kwangbong Lee ${ }^{2}$ \\ ${ }^{1}$ Department of Business, Hunan Information Science Vocational College \\ ${ }^{2}$ Department of International Trade, Inje University \\ Received 16 January 2012, revised 12 February 2012, accepted 17 February 2012
}

\begin{abstract}
Weather derivatives designed to manage casual changes of weather, as opposed to catastrophic risks of weather, are relatively a new class of financial instruments. There are still many theoretical and practical challenges to the effective use of these instruments. The objective of this paper is to develop a pricing approach for valuing weather derivatives and presents a case study that is practical enough to be used by the risk managers of electrical utility firms. Utilizing daily average temperature data of Guangzhou, China from $1^{\text {st }}$ January 1978 to $31^{\text {st }}$ December 2010, this paper adopted a univariate time series model to describe weather behavior dynamics and calculates equilibrium prices for weather futures and options for an electrical utility firm in the region. The results imply that the risk premium is an important part of derivatives prices and the market price of risk affects option values much more than forward prices. It also demonstrates that weather innovation as well as weather risk management significantly affect the utility's financial outcomes.
\end{abstract}

Keywords: Incomplete markets, risk management, weather derivatives.

\section{Introduction}

The economic impact of weather on many commercial and recreational activities is significant and varies both geographically and seasonally. Similar to the effects of environmental management on firm performance (Kang et al., 2011), weather risk management is now an essential function for any risk manager.

Especially, Chinese energy firms operating under rapidly deregulated environment now come to realize intensively the effect of weather on their operations. In the early days of deregulation, energy firms started to note weather risks, tried to quantify them, and looked to the insurance industry for risk solutions. The insurance industry, however, was not then and is still not receptive to requests for such non-catastrophic protection in nature. Accordingly, the energy industry was left to fend for itself.

${ }^{1}$ Lecturer, Department of Business, Hunan Information Science Vocational College, Changsha 410151, China.

2 Corresponding author: Professor, School of Economics \& International Trade, Inje University, Gimhae 621-749, Korea. E mail: tradlkb@inje.ac.kr 
The underlying variables for weather contracts are numerous. Temperature, rainfall, snowfall, and precipitation are just a few of them. Even Promethium 10 concentration (Lee, 2010) and ozone concentration (Lee, 2009) are all weather related. However, it is estimated that over $90 \%$ of the weather derivatives now actively traded are based on Cooling Degree Days $(C D D)$ and Heating Degree Days $(H D D)$. The number of $C D D$ and $H D D$ for a specific day is the number of degrees that the temperature deviates from a defined baseline level. The $C D D$ and $H D D$ index futures are legally binding agreements to buy or sell the value of $C D D$ and $H D D$ index at a certain time in the future.

The weather derivatives market is a classic example of an incomplete market because the underlying temperature itself is not a tradable commodity (Alaton et al., 2002; Calum, 2005). Therefore, it is not possible to apply the usual risk-neutral assumption or a perfect replication of the weather derivatives payoff.

The pricing mechanisms suggested in the literature (Alaton et al., 2002; Benth and Benth, 2005; Campbell and Diebold, 2002; Cao and Wei, 2004) are various, burn analysis, index modeling approaches, daily weather variable process models, and the so called daily simulation approaches to name a few. Burn analysis valuates a derivative simply by averaging all payoffs that would have been realized in the past. Index modeling goes one step further and models the weather index with a probability distribution of which parameters are usually estimated based upon historical data. The actual pricing then proceeds by taking the expected value of the derivatives' payoff plus a possible risk premium.

In a discrete time framework, Cao and Wei (1999) suggest an equilibrium pricing model based on the Euler equation and the fact that in equilibrium both the financial market and the goods market are clear. Similar in a sense to Cao and Wei (1999), Shim and Hwang (2011) forecast volatility via conditional autoregressive value at risk model on the basis of support vector quantile regression. Benth and Benth (2005) follow up Cao and Wei (1999) with a detailed model for the temperature dynamics. Since weather factors are not assets and can not be traded, the traditional no-arbitrage approach, risk-neutral valuation is not directly applicable to weather derivatives.

The main purpose of this paper is to develop a pricing approach for valuing weather derivatives and presents a case study that is practical enough to be used by the risk managers of electrical utility firms. The paper is organized as follows. The Section 2 describes the data used and defines the stochastic temperature behaviors. The equilibrium pricing approach is presented and its application procedures are discussed in the Section 3. The Section 4 designs a scenario and estimates the market price of risk. A case study for a Guangzhou electrical utility is presented and its hedging effectiveness is analyzed in the Section 5. The final section concludes.

\section{Data and the estimation of temperature behaviors}

In order to model the temperature variable, historical daily temperature data, covering the period from January $1^{\text {st }}$, 1978, to December $31^{s t}$, 2009 for Guangzhou, is obtained from the China Meteorological Data Sharing Service System (CMDSSS), a subsidiary of the China Meteorological Administration (CMA). The 2010's data is saved for the purpose of evaluation.

Pricing temperature derivatives requires modeling and forecasting temperature behaviors. According to Campbell and Diebold (2002), the daily temperature dynamics should include 
three fundamental components: trends, seasonality, and cyclical volatility. The trend component reflects the global warming tendency, which is likely minor but relevant. Seasonal character is very important part in any model fit to daily average temperature, as daily temperature pronounced seasonal variation, with both the amplitude and seasonal patterns. The seasonality exists both in the mean and variance. The cycle factor means any sort of persistent dynamics apart from seasonality and trend, which can be captured by lagged volatility. In order to capture the three features of the daily average temperature, the temperature processes $Y_{t}$ can be expressed as follows:

$$
Y_{t}=\text { Trend }_{t}+\text { Seasonal }_{t}+\sum_{l=1}^{L} \rho_{t-l} Y_{t-l}+\sigma_{t} \xi_{t}, \quad \xi_{t} \stackrel{i i d}{\sim} N(0,1)
$$

where

$$
\begin{gathered}
\text { Trend }_{t}=\beta_{0}+\beta_{1} t \\
\text { Seasonal }_{t}=\sum_{p=1}^{P}\left[\delta_{p}^{c} \cos \left(2 \pi p \frac{d(t)}{365}\right)+\delta_{p}^{s} \sin \left(2 \pi p \frac{d(t)}{365}\right)\right] \\
\sigma_{t}^{2}=\sum_{q=1}^{Q}\left[\delta_{q}^{c} \cos \left(2 \pi q \frac{d(t)}{365}\right)+\delta_{q}^{s} \sin \left(2 \pi q \frac{d(t)}{365}\right)\right]+\sum_{r=1}^{R} \alpha_{r} \sigma_{t-r}^{2} \xi_{t-r}^{2}
\end{gathered}
$$

$\rho$ represents the parameters of temperature autoregressive lags to be estimated, $\xi_{t}$ is the random variable of the temperature, $\alpha$ is the parameter of GARCH term, $d(t)=1,2,3, \cdots, 365$, representing the dates throughout a year. $L$ captures long memory dynamics of the daily temperature. $P$ and $Q$ captures the seasonal cycle, and $R$ captures the GARCH effects of the volatility. According to the minimum Akaike and Schwarz criteria, Campbell and Diebold (2005) suggested the adequate value for these parameters. The setting of $L=25, P=3, Q=$ 3 and $R=1$ has been employed.

Almost all parameters in Table 2.1 are estimated with very low standard errors, implying the proper specification of the temperature process. The warm trending parameter is very low, equals 0.000313 , which indicates the temperature has only risen by about $0.37^{\circ} \mathrm{C}$ during the past 32 years. The estimated autoregressive coefficients display an interesting pattern. While the first order autoregressive behavior tends to be stronger and has the value up to 0.935 , coefficients on subsequent lags become significantly negative before decaying. The coefficient on the second autoregressive lag, for example, is typically about -0.275 . And after 3 lags, the autoregressive behavior performs insignificantly. Roughly speaking, a stronger autocorrelation means less dramatic changes in temperature.

\section{Pricing weather derivatives}

To obtain the market price of risk attached to the weather variable, the extended Lucas (1978) pure exchange economy model proposed by Cao and Wei (2004) is employed. The 
Table 2.1 Estimation results of daily average temperature (1978-2009)

\begin{tabular}{cccccccccc}
\hline & $\beta_{0}$ & $\beta_{1}$ & $\delta_{1}^{c}$ & $\delta_{2}^{c}$ & $\delta_{3}^{c}$ & $\delta_{1}^{s}$ & $\delta_{2}^{s}$ & $\delta_{3}^{s}$ & $\rho_{-1}$ \\
\hline Coefficient & 6.6891 & $3.1 \mathrm{E}-05$ & -1.952 & -0.2449 & -0.0136 & -0.8175 & -0.1533 & 0.0050 & 0.9354 \\
Std.Error & 0.3445 & $5.0 \mathrm{E}-06$ & 0.0874 & 0.0271 & 0.0256 & 0.0708 & 0.0285 & 0.0246 & 0.0085 \\
Z-Statistic & 19.413 & 6.162 & -22.342 & -9.091 & -0.529 & -11.563 & -5.418 & 0.1199 & 110.42 \\
\hline & $\rho_{-2}$ & $\rho_{-3}$ & $\rho_{-4}$ & $\rho_{-5}$ & $\rho_{-6}$ & $\rho_{-7}$ & $\rho_{-8}$ & $\rho_{-9}$ & $\rho_{-10}$ \\
\hline Coefficient & -0.2754 & 0.0534 & -0.0162 & 0.0147 & 0.0029 & 0.0104 & -0.0003 & -0.0374 & 0.0464 \\
Std.Error & 0.0119 & 0.0127 & 0.0129 & 0.0130 & 0.0128 & 0.0128 & 0.0129 & 0.0129 & 0.0129 \\
Z-Statistic & -23.130 & 4.0988 & -1.1604 & 1.1686 & 0.2258 & 0.8488 & -0.0312 & -2.7991 & 3.6417 \\
\hline & $\rho_{-11}$ & $\rho_{-12}$ & $\rho_{-13}$ & $\rho_{-14}$ & $\rho_{-15}$ & $\rho_{-16}$ & $\rho_{-17}$ & $\rho_{-18}$ & $\rho_{-19}$ \\
\hline Coefficient & -0.0011 & -0.0089 & -0.0016 & -0.0127 & 0.0238 & -0.0050 & -0.0026 & -0.0093 & 0.0065 \\
Std.Error & 0.0129 & 0.0126 & 0.0126 & 0.0130 & 0.0128 & 0.0128 & 0.0128 & 0.0127 & 0.0127 \\
Z-Statistic & -0.2691 & -0.7539 & -0.1615 & -1.0237 & 1.935 & -0.2981 & -0.2273 & -0.730 & 0.4163 \\
\hline & $\rho_{-20}$ & $\rho_{-21}$ & $\rho_{-22}$ & $\rho_{-23}$ & $\rho_{-24}$ & $\rho_{-25}$ & $\sigma_{t}$ & $\delta_{c, q 1}$ & $\delta_{c, q 2}$ \\
\hline Coefficient & 0.0028 & -0.0061 & 0.0003 & -0.0055 & 0.0269 & -0.0220 & 2.659 & 0.8761 & -0.1109 \\
Std.Error & 0.0128 & 0.0129 & 0.0129 & 0.0124 & 0.0121 & 0.0090 & 0.0322 & 0.0332 & -0.0288 \\
Z-Statistic & 0.2519 & -0.4562 & 0.0473 & -0.4145 & 2.2456 & -2.4515 & 69.099 & 26.386 & 3.8558 \\
\hline & $\delta_{c, q 3}$ & $\delta_{s, q 1}$ & $\delta_{s, q 2}$ & $\delta_{s, q 3}$ & $\alpha_{1}$ & $R^{2}$ & $R^{2}$ & & \\
\hline Coefficient & -0.0686 & 0.9250 & 0.2465 & -0.0828 & 0.3724 & 0.9104 & 09101 & & \\
Std.Error & 0.0359 & 0.0314 & 0.0331 & 0.0303 & 0.0191 & & & & \\
Z-Statistic & -1.9073 & 29.4392 & 7.4462 & 2.7332 & 19.478 & & & & \\
\hline
\end{tabular}

main assumption is that the fundamental uncertainties in the economy are driven by the aggregate dividend $\delta(t)$ and the temperature $Y(t)$.

Then, the risk premium for temperature contracts can be calculated by the equilibrium pricing method. Aggregate dividends can be viewed as the dividends on market portfolio. For a representative investor, the complete standard equilibrium conditions imply that total consumption is equal to the aggregate dividends generated from the risky portfolio. Therefore, the time t price of a contingent claim with a payoff $q_{T}$ at a future time $T$, denoted by $X_{t}(T)$ is:

$$
X_{t}(T)=\frac{1}{U_{c}\left(\delta_{t}, t\right)} E_{t}\left(U_{c}\left(\delta_{T}, T\right)\right) q_{T}, \forall t \in(0, T)
$$

where $c_{(\cdot, \cdot)}$ denotes the consumption function, $U_{c}\left(\delta_{t}, T\right)$ is the first derivative of the period- $T$ utility function on consumption, $\delta_{t}$. This shows that the present values of contingent claims can be determined by an agent's preference, which may relate to both aggregate dividends and temperature factors involved in the dividend process. The representative agent is usually assumed to have a risk preference characterized by relative risk aversion in the literature.

The representative agent's period utility function can be described by a power form:

$$
U_{t}\left(\delta_{t}\right)=e^{-\rho \times t} \frac{\rho_{t}^{\gamma+1}}{\gamma+1}
$$

where $\delta_{t}$ is the aggregate dividend, which equals the aggregate consumption under the Lucas pure exchange economy. $\rho$ is the rate of time preference, which tell us how much the investor values the present relative to the future. And the risk parameter $\gamma \in(-\infty, 0]$ is to perform a risk aversion behavior, and its value represents the degree of investor's risk aversion. $\gamma=0$ corresponds to the risk neutral case. 
The dividend process is assumed to follow an autoregressive process, where current aggregate dividend $\delta_{t}$, is dependent on the dividend in the previous period $\delta_{t-I}$, evolving the following Markov process:

$$
\ln \delta_{t}=\alpha+\mu \ln \delta_{t-I}+\nu_{t}, \forall \mu \leq 1
$$

where $\alpha$ is the constant and can be interpreted as the growth rate of dividends , 1- $\mu$ measures the speed of mean reversion, and the error term takes the following form:

$$
\begin{aligned}
& \nu_{t}=\rho \epsilon_{t}+\sigma\left[\frac{\phi}{\sqrt{1-\phi^{2}}} \xi_{t}+\eta_{1} \xi_{t-1}+\eta_{2} \xi_{t-2}+\eta_{3} \xi_{t-3}+\cdots++\eta_{m} \xi_{t-m}\right], \\
& \xi_{t} \stackrel{i i d}{\sim} N(0,1)
\end{aligned}
$$

In the above, $\epsilon_{t}$ is an i.i.d., standard normal variable which measures the randomness due to all factors other than the temperature uncertainty; and $\xi_{t}, \forall t$ represents the temperature variable and its lagged terms innovations.

If the equilibrium price of a riskless pure discount bond paying 1 unit of consumption goods as maturity time $T$, meaning $q_{t}=1$, the contingent claim at time $t$ can be viewed as a discount factor with maturity $T$. Then, under the constant relative risk aversion (CRRA) utility in Equation (3.2), and the dividend process in Equation(3.3) and (3.5), the price of a pure discount bond at time $t$ with maturity $T$ can be determined by:

$$
\begin{aligned}
D_{t} t & =\frac{e^{-\rho(T-t)} E_{t}\left(\delta_{t}^{r}\right)}{\delta_{t}^{\gamma}} \\
& =\exp \left\{-\rho(T-t)+\gamma\left(\mu^{T-t}-1\right) \ln \delta_{t}+\alpha \gamma \sum_{i=t+1}^{T} \mu^{T-i}+\frac{1}{2} \times \frac{\gamma^{2} \sigma^{2}}{1-\phi^{2}} \sum_{i=t+1}^{T} \mu^{2(T-i)}\right\}
\end{aligned}
$$

Considering the requirement of the Chinese State Council that for all public buildings, the summer temperature limit for air conditioning should not be lower than $26^{\circ} \mathrm{C}$, the baseline temperature is set in this paper as $25^{\circ} \mathrm{C}$. Then,

$$
C D D\left(T_{1}, T_{2}\right)=\sum_{i=T_{1}}^{T_{2}} \max \left(Y_{i}-25,0\right) .
$$

Assume a $C D D$ forward contract with a tick size of $¥ 1$ and delivery price $P$ and the contract period starting at $T_{1}$ and ending at maturity $T_{2}\left(T_{2}>T_{1}\right)$. The value of $P$ will be the forward price at time $t$ :

$$
F_{C D D}\left(T_{1}, T_{2}, P\right)=\frac{E_{t}\left(\delta_{T_{2}}^{\gamma} C D D\left(T_{1}, T_{2}\right)\right)}{E_{t}\left(\delta_{T_{2}}^{\gamma}\right)}=\frac{e^{-\rho\left(T_{2}-t\right)} \delta_{t}^{-\gamma} E_{t}\left(\delta_{T_{2}}^{\gamma} C D D\left(T_{1}, T_{2}\right)\right)}{d_{t}\left(T_{2}\right)}
$$

An European option written on $C D D\left(T_{1}, T_{2}\right)$ with a maturity $T_{2}$ and a strike price $A$ is derived in a similar way. Denoting the call and put prices at time $t$ as $C_{C D D}\left(T_{1}, T_{2}, A\right)$ and $P_{C D D}\left(T_{1}, T_{2}, A\right)$, respectively, the call and put option values are priced as

$$
\begin{array}{r}
\left.\left.C_{C D D}\left(T_{1}, T_{2}, p\right)=e^{-\rho\left(T_{2}-t\right)} \delta_{t}^{-\gamma} E_{t}\left(\delta_{T_{2}}^{\gamma} \max \left(C D D\left(T_{1}, T_{2}\right)\right)-A\right), 0\right)\right) \\
P_{C D D}\left(T_{1}, T_{2}, p\right)=e^{-\rho\left(T_{2}-t\right)} \delta_{t}^{-\gamma} E_{t}\left(\delta_{T_{2}}^{\gamma} \max \left(A-C D D\left(T_{1}, T_{2}\right), 0\right)\right)
\end{array}
$$




\section{The estimation of market price of risk}

The model setup calls for a joint estimation of the aggregate dividend process and the daily temperature behavior. Monte Carlo simulation is employed to generate the temperature and aggregate dividend process jointly, and performed for the specific scenarios under different risk aversion and dividend process parameters.

Assumptions are made as follows:

First, the preference of the time $\rho$ is set at 0.03 , which is the annual risk free rate according to the People's Bank of China in February 2011.

Second, based on the empirical findings in the literature, the mean reversion parameter $\mu$ is suggested to be 0.9 and $\sigma$ equals 0.2 .

Third, Zou (2011) derived the contemporaneous correlation coefficient of 0.28 between the temperature and dividend processes from the equations (3.3) and (3.4) utilizing the 32 years' monthly average temperature in Guangzhou and the city's monthly power consumption. The contemporaneous correlation coefficient $\Phi$ is set as \pm 0.3 .

Fourth, since the joint solutions of $\sigma$ and $\delta_{t}$ are not unique and negatively related with the fixed yields of the pure discount bond, the average dividend growth rate $\sigma$ and the initial dividend $\delta_{t}$ can be set according to $e^{-r(t, T)(T-t)}=E_{t}\left(U_{c}\left(\delta_{T}\right) / U_{c}\left(\delta_{t}\right)\right)$.

Finally, we let risk aversion parameter $\gamma$ be a comparative static variable. Since most empirical studies indicate that $\gamma$ ranges form zero to -2.0 , three cases are examined: $\gamma$ equals to $-0.5,-1.0$ and -2.0 . Here, we do not consider the so-called equity premium puzzle, which indicates $\gamma<-2.0$. For each risk aversion scenario, three correlation levels are examined, each of them can be positive or negative.

Thus, given the assumptions, the specifications for the representative agent's preference in Equation (3.2), the dividend process in Equation (3.3) and (3.4), and temperature dynamics in Equations (2.1), together with the general pricing equation in Equation (3.1), enable the valuation of any claim contingent upon the temperature variable. The forward prices for the $C D D$ contracts covering the period of July 1st to September 30th are presented in Table 4.1 .

The numbers in the parentheses are the percentage difference from the risk-neutral price which equals 538.40 derived from the simulation, and are defined as the risk premium. With a given correlation, the value of the risk premium will get larger as the value of $\gamma$ increases. The largest percentage price difference, around $2.45 \%$, appears when $\gamma=-2.0$ and $\Phi=-0.3$. It implies that when the degree of an agent's risk aversion increases, they will ask for more risk premiums. And the prices of $C D D$ forwards are higher than risk-neutral price when the correlation parameter $\Phi$ equals -0.3 and lower when the correlation parameter $\Phi$ equals 0.3 .

Table 4.1 Seasonal $C D D$ forward prices

\begin{tabular}{|c|c|c|c|}
\hline Items & $\gamma=-0.5$ & $\gamma=-1.0$ & $\gamma=-2.0$ \\
\hline$\Phi=-0.3$ & $545.585(1.33 \%)$ & $547.1998(1.63 \%)$ & $551.5996(2.45 \%)$ \\
\hline$\Phi=0.3$ & $532.215(-1.15 \%)$ & $529.6002(-1.45 \%)$ & $526.2004(-2.27 \%)$ \\
\hline
\end{tabular}

The prices of seasonal $C D D$ call and put options are also valued for the same setting and the results are illustrated in Table 4.2. For convenience, the strike price is set to equal to the risk-neutral forward price so that the risk-neutral call and put options are exactly at 
the money and have the same values. Thus, the risk-neutral option values can be calculated by setting the correlation between the temperature and the aggregate dividend processes as zero. The results are similar to the forward prices. However, the risk premiums in option prices are larger than those in the forward prices. The largest percentage price differences are $-14.02 \%$ for call and $22.25 \%$ for put when $\gamma=-2.0$ and $\Phi=-0.3$. It means that the $C D D$ call (put) option contains a risk premium of $-14.02 \%$ (22.25\%) purely due to the market price of risk associated with temperature variable.

The results are consistent with the results of Cao and Wei (2004), and Chang (2009). When the correlation parameter $\Phi<0, C D D$ option prices are higher than those under the risk-neutral world. The results imply that in the case of a negative (positive) correlation, a higher risk aversion $(\gamma=-2.0)$ will lead to higher (lower) option prices. The explanation of this result is rather complicated. If we look at the pricing equation for $C D D$ call options, we can see that the dividend ratio $\delta_{T_{2}} / \delta_{T}$ is raised to the power of $\gamma$, and is negative by definition, a higher dividend ratio will turn to a lower price. When a negative correlation is expected, a higher $C D D$ accumulated value will go together with a lower dividend $\delta_{T_{2}}$, and thereby a lower dividend ratio. The more risk-averse the preferences are the larger will the above effect be. It means that for the given negative correlation $(\Phi=-0.3)$, the price gets higher as the risk-aversion increases. The reverse argument holds when $\Phi=0.3$. This is also an explanation why the option price decreases when the correlation increases for a given $\gamma$. For the case of puts, the effect is opposite.

Table 4.2 Seasonal $C D D$ option prices

\begin{tabular}{ccccc}
\hline \hline Items & \multicolumn{1}{c}{$\gamma=-0.5$} & $\gamma=-1.0$ & $\gamma=-2.0$ \\
\hline \multirow{2}{*}{$\Phi=-0.3$} & Call & $38.7919(7.4 \%)$ & $39.4048(9.09 \%)$ & $41.0746(13.72 \%)$ \\
\cline { 2 - 5 } & Put & $31.9618(-11.51 \%)$ & $31.9618(-11.51 \%)$ & $28.3638(-21.47 \%)$ \\
\hline \multirow{2}{*}{$\Phi=0.3$} & Call & $33.3383(-7.7 \%)$ & $32.7255(-9.4 \%)$ & $31.0556(-14.02 \%)$ \\
\cline { 2 - 5 } & Put & $40.558(12.28 \%)$ & $41.524(14.96 \%)$ & $44.156(22.25 \%)$ \\
\hline
\end{tabular}

\section{Actual hedging effectiveness}

There has existed a great deal of studies about using weather derivatives to hedge weather risk in energy markets. Since weather derivatives are a hedge on volume, not on price, some care is necessary in building a net position regarding a physical commodity such as power.

Facing summer months, risk managers at Guangzhou electrical utility, the firm in the case study, anticipate being short of generation and explore the cost of protection against abnormal hot summer. To determine the timing of financing needs, risk managers are expected to consider both the utility's weather exposure and production capacity (Ellithorpe and Putnam, 2000). Duan and Qian (2009) showed that during summer seasons, July to September, with $C D D$ up to 500 , the power consumption of Guangzhou is satisfied at the maximum production capacity of 100 billion KWH, and its revenues are 6 billion RMB.

In summer days, at the temperature higher than $25^{\circ} \mathrm{C}$, people begin to turn on their air conditioners to cool interior spaces. According to Duan and Qian (2009), the correlation between the electrical power consumption of Guangzhou City and the $C D D$ value is 0.65 during the summer season. More specifically, if the $C D D$ value increases $1 \%$, the power consumption of Guangzhou city will increase $0.65 \%$. 
If the $C D D$ value is greater than 500 , power consumption begins to exceed the utility's generation capacity and the utility will have to purchase incremental power in the wholesale spot market at prices that are potentially much higher than the price it charges currently to its customers. Since per $C D D$ above 500 will lead to an increasing power consumption of $130,000,000 \mathrm{KWH}$, assuming the cost for this incremental power of $0.10 \mathrm{RMB}$ per $\mathrm{KWH}$, then the loss caused by one excess $C D D$ is $13,000,000(130,000,000 \times 0.10)$ RMB.

Based on the foregoing information, one solution for the utility would be to purchase call option. The terms of the structure are summarized in Table 5.1. Since the notional payout for each $C D D$ is $20,000 \mathrm{RMB}$ and the lose exposure is 13 million RMB, the utility needs 650 shares of contracts.

As mentioned above, the contract price consists of the two components: a riskless component that corresponds to the known dividends during the life of the option and a risk component. Except for the strike price of $500 C D D$ and the season $C D D$ value of 538.4 from the simulation of the temperature model, other parameters are the same. Then the price of this $C D D$ contract can be calculated as the equation (3.7).

Table 5.1 $C D D$ call option for Guangzhou electrical utility

\begin{tabular}{ll}
\hline \hline Contract Parameters & Value or Items \\
\hline Type & Call option \\
Current Time & December 31, 2009 \\
Location & Guangzhou, Weather Station ID: \#59287 \\
Index & $C D D$ measured at the location \\
Calculation Period & July 1, 2010 to September 30, 2010 \\
Attachment Strike & $500 C D D$ accumulated during calculation period \\
Notional Payout Rate & 20,000 RMB per $C D D$ above the attachment \\
Limit of pay out & RMB 1M \\
\hline
\end{tabular}

Utilizing the contemporaneous correlation of 0.3 for the summer season as before, the prices of the $C D D$ call option in Table 5.2 are around 53.00 under different risk aversion parameters, and the prices are the amount that the electrical utility is willing to pay to protect its revenues.

Table 5.2 CDD Call option prices for Guangzhou electrical utility

\begin{tabular}{cccc}
\hline \hline Items & $\gamma=-0.5$ & $\gamma=-1.0$ & $\gamma=-2.0$ \\
\hline$\Phi=-0.3$ & 52.20 & 52.12 & 51.90 \\
$\Phi=0.3$ & 52.92 & 53.00 & 53.22 \\
\hline
\end{tabular}

The specified $C D D$ forward value of 538.4 is regarded as the anticipated value for 2010 . With the given capacity, the electrical utility will buy 650 shares of contracts for each $C D D$ more than 500 to smoothen its revenue. To protect against incremental $38 C D D$ s, the utility need to buy $24,700(38 \times 650)$ shares of contracts. Since the premium for each share of contracts is $¥ 53.00$, for the expected $38 C D D$ s, the call option premium will be $¥ 1,309,100$ $(650 \times 53.00 \times 38)$. As the option contract gets matured, its actual $C D D$ value reaches 565 . The payout pattern for the transaction is summarized in Figure 5.1. The cost of the utility without any active risk management measure is shown as dot $A$ and equals $845(65 \times 13)$ million RMB. Through the purchase of call options, the utility will be compensated because of the abnormal hot summer. Then its operating cost is shown as dot $B$ and equals 196.3 


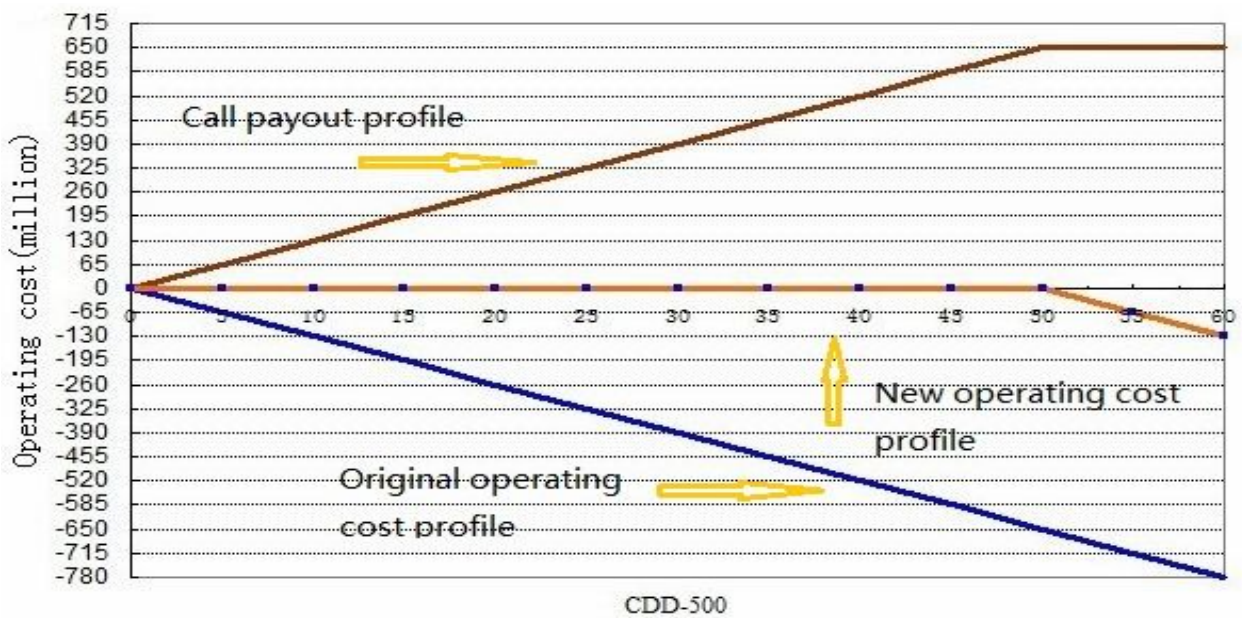

Figure 5.1 The effects of call option for Guangzhou electrical utility

million, 650 million of option payout at $565 C D D$ minus 845 million of utility's loss at 565 $C D D$ minus 1.3 million of premium payments. In this fashion, the utility hedges against the severe economic loss that would be incurred in the abnormal hot summer. While the hedging procedure has been described for an electrical utility firm, the problem of effective hedging against weather risk is not limited to the energy industry.

\section{Conclusions}

Applying the Campbell and Diebold (2002)'s time series model and Cao and Wei (2004)'s extended Lucas (1978) pure exchange economy model to the 32 years' temperature data in the city of Guangzhou, China, this paper derived the equilibrium prices for $C D D$ forward, call and put options. The estimation of the market price of risk over a range of risk aversion parameters implies that the risk premium can be an important part of derivatives price and the market price of risk affects options much more than forwards. Consequently, in the cases that the risk-neutral valuation is not directly applicable as in this study, the assumption of zero market price of risk could result in significant pricing errors.

The case study for Guangzhou electrical utility demonstrates that weather innovation could significantly affect the utility's outcomes and that risk management meaningfully influences its financial conditions. Although the energy industries happen to be the first to embrace the weather market, other industries of which cash flows have fluctuated with changing weather conditions are likely to use weather derivatives once they become available. There are still many theoretical and practical challenges to the effective use of weather derivatives. The derivation of more accurate models for dynamic weather behaviors and the market price of risk would facilitate broader acceptance of weather derivatives among other industries and eventually foster overall market growth. 


\section{References}

Alaton, P., Djehiche, B. and Stillberger, D. (2002). On modelling and pricing weather derivatives. Applied Mathematical Finance, 9, 1-20.

Benth, F. E. and Benth, J. S. (2005). The volatility of temperature and pricing of weather derivatives. Pure Mathematics, 12, 1-17.

Calum G. T. (2005). The pricing of degree-day weather options. Agricultural Finance Review, 65, 59-85.

Campbell, S. D. and Diebold, F. X. (2002). Weather forecasting for weather derivatives, PIER working paper. No. 02-046.

Campbell, S. D. and Diebold, F. X. (2005). Weather forecasting for weather derivatives. Journal of American Statistical Association, 100, 6-16.

Cao, M. and Wei, J. Z. (1999). Pricing weather derivative: An equilibrium approach, Working Paper Series. Available at: http://ssrn.com/abstract $=172414$.

Cao, M. and Wei, J. Z. (2004). Weather derivatives valuation and market price of weather risk. The Journal of Futures Markets, 24, 1065-1089.

Chang, C. C., Lin, J. B. and Shen, W. M. (2009). Pricing weather derivatives using a predicting power time series process. Asia-Pacific Journal of Financial Studies, 38, 863-890.

Duan, H. and Qian, H. (2009). Responses of electric power consumption to climate change in Guangzhou city. Journal of Applied Meteorological Science, 20, 80-87.

Ellithorpe, D. and Putnam, S. (2000). Weather derivatives and their implications for power markets. Journal of Risk and Finance, 1, 19-28.

Kang, M. S., Choi, H. S. and Park, B. C. (2011). Effects of environmental management on firm performance. Journal of the Korean Data 8 Information Science Society, 22, 523-536.

Lee, H. (2009). Analysis of statistical models for ozone concentrations at the Paju city in Korea. Journal of the Korean Data \& Information Science Society, 20, 1085-1092.

Lee, H. (2010). Analysis of time series models for PM10 concentrations at the Suwon city in Korea. Journal of the Korean Data $\mathbb{E}$ Information Science Society, 21, 1117-1124.

Lucas, R. E. Jr. (1978). Asset prices in an exchange economy. Econometrica, 46, 1429-1445.

Shim, J. and Hwang, C. (2011). Forecasting volatility via conditional autoregressive value at risk model based on support vector quantile regression. Journal of the Korean Data \& Information Science Society, 22, 589-596

Zou, Z. X. (2011). Weather derivatives pricing: An application to Guangzhou electrical utility in China, $\mathrm{Ph}$. D. Thesis Department of International Trade. Inje University. 